\title{
Téoros
}

Revue de recherche en tourisme

\section{Entretien avec Stefan Roesch, consultant exécutif pour le Market Research Institute CenTouris (Bavière, Allemagne)}

\section{Ari Virtanen}

Volume 30, numéro 1, 2011

URI : https://id.erudit.org/iderudit/1012120ar

DOI : https://doi.org/10.7202/1012120ar

Aller au sommaire du numéro

Éditeur(s)

Université du Québec à Montréal

ISSN

0712-8657 (imprimé)

1923-2705 (numérique)

Découvrir la revue

Citer ce document

Virtanen, A. (2011). Entretien avec Stefan Roesch, consultant exécutif pour le Market Research Institute CenTouris (Bavière, Allemagne). Téoros, 30(1),

150-151. https://doi.org/10.7202/1012120ar d'utilisation que vous pouvez consulter en ligne. 


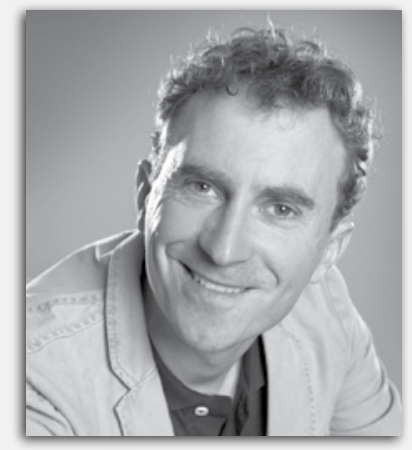

\title{
Entretien
}

\author{
avec Stefan ROESCH, consultant exécutif \\ pour le Market Research Institute CenTouris \\ (Bavière, Allemagne)
}

\author{
Propos recueillis par Ari VIRTANEN, adjoint à la rédaction
}

Stefan Roesch est consultant en étude de marché et en ciné-tourisme. II est impliqué dans ce dernier domaine depuis 2004. Stefan Roesch est aussi auteur de l'ouvrage The Experiences of Film Location Tourists (2009, recensé dans ce numéro).

Stefan Roesch a complété ses études de maîtrise en géographie du tourisme. II détient un doctorat de l'Université d'Otago, en NouvelleZélande. Avant de rédiger sa thèse, il a travaillé en gestion du tourisme en Bavière. II occupe présentement un poste de consultant principal pour la firme Market Research Institute CenTouris, en Bavière.

\section{Comment devient-on consultant en ciné-tourisme?}

Tout a commencé avec la thèse que j'ai écrite sur le ciné-tourisme international, où je présente des études de cas sur The Lord of the Rings en Nouvelle-Zélande, Star Wars en Tunisie et The Sound of Music en Autriche. J'ai continué la recherche sur le sujet même après mes études. Faisant appel à mon réseau croissant de contacts à travers le monde, j'ai commencé à faire de la consultation pour des offices de cinéma et des gestionnaires en tourisme en relation avec les opportunités de développement en ciné-tourisme. Je dois préciser que mon travail comme consultant en ciné-tourisme se fait à la pige. Je suis avant toute chose consultant exécutif en études de marché, en marketing et en développement commercial.

\section{Parlez-nous un peu de la nature même de votre travail comme consultant en matière de ciné-tourisme.}

La principale partie de mon travail en ciné-tourisme consiste à participer à des conférences organisées par l'industrie afin de sensibiliser les gestionnaires en tourisme et les offices de cinéma aux possibilités du ciné-tourisme. J'aide aussi les destinations à développer des stratégies de marketing autour des films pour créer des produits de ciné-tourisme, comme des tours guidés des sites ayant servis lors de tournages.

L'un des projets sur lesquels j'ai travaillé, par exemple, consistait à aider l'organisme autrichien Wilder Kaiser à développer des outils d'intérêt autour de la télésérie The Mountain Doctor. Cela s'est fait par le développement d'un forfait d'une semaine basée sur la production en question, par le développement d'un microsite lié à la production, par un blogue pour le décor ainsi que par l'embauche de l'acteur principal pour recommander la destination.

Même si la relation entre le tourisme et le cinéma n'est pas nouvelle, le ciné-tourisme ne semble être devenu un véritable phénomène que tout récemment. Est-ce que cette lecture est bonne ou avons-nous cette impression juste parce que les universitaires ne font que commencer à y porter attention?
Le ciné-tourisme n'est pas un nouveau phénomène. Prenez le cas de films comme The Third Man (1949), Niagara (1953), To Catch a Thief (1955), Bridge on the River Kwai (1958), Lawrence of Arabia (1962) et The Sound of Music (1965) : voilà de très beaux exemples de films qui génèrent du ciné-tourisme. Ce qui a changé depuis les deux dernières décennies est le fait que de plus en plus de films à grands budgets sont tournés hors studios, sur des sites exotiques, qu'au même moment le tourisme devient un phénomène de masse mondial et que l'attention des médias se porte désormais sur le phénomène du ciné-tourisme. Les universitaires ont simplement bondi sur le train en ce sens où ils ont découvert un nouveau champ de recherche (en commençant par les chercheurs américains Riley et Van Doren au début des années 1990).

Par les cas rapportés dans la littérature, il semble que de plus en plus de promoteurs misent sur le ciné-tourisme pour développer leurs destinations. On voit aussi que certaines destinations semblent tirer un plus grand bénéfice d'une production dans le temps que d'autres ciné-destinations. Quels facteurs vont contribuer au succès à long terme d'une ciné-destination? Le problème est qu'il n'y a pas deux films ou séries télévisées qui soient semblables. Le succès dans la durabilité du ciné-tourisme est donc lié aux productions individuelles, comme la qualité du scénario, la qualité de la distribution (les acteurs), la qualité des sites employés, le type de film, etc. II est vrai que si la destination illustrée dans le film réussit à développer sa propre stratégie pour créer des produits de ciné-tourisme, alors elle peut influencer favorablement la longévité du cycle de vie du ciné-tourisme. Comme mentionné plus tôt, de tels produits nécessitent d'être développés avec professionnalisme et d'être bien présentés. Ce qui aide c'est la mise en place de réseaux de communication entre les différents partenaires (les offices du cinéma, les autres organisations impliquées en tourisme et la compagnie de production elle-même).

Les ciné-touristes ont-ils des besoins particuliers en comparaison avec les touristes plus "conventionnels»? L'aspect le plus important qu'il faut à garder en tête, lorsqu'il est question de ciné-touristes, c'est qu'il faut leur fournir une expérience chargée d'émotions qui rende le site bien vivant. Cela n'est possible qu'avec l'aide d'un guide sur le terrain. Le guide doit pouvoir montrer quels emplacements ont été utilisés dans les scènes, raconter des détails à propos du tournage en plus des commentaires plus généraux sur le lieu. Voilà ce qui rend la visite vivante. Par conséquent, les guides de lieux de tournages jouent un rôle clé dans un produit de ciné-tourisme et doivent ainsi être de grands connaisseurs, même à propos du film visé. 
Le ciné-tourisme propose, entre autres, la visite de lieux historiques. Y a-t-il une possibilité de contradiction quand un lieu historique, par exemple, devient l'objet d'intérêt à travers le regard du ciné-tourisme?

La question varie selon le film spécifique tourné dans une région. Si le film fait appel à des édifices historiques qui existent depuis déjà un certain temps, et qui continueront d'exister dans l'avenir, il est évident que les ciné-touristes vont tenter de visiter ces édifices. II y a assez de preuves quant aux problèmes qu'engendre le surencombrement les lieux. Un bon exemple à cet effet serait la chapelle Rosslyn, en Écosse, employée dans le film The Da Vinci Code et qui est subséquemment passée de lieu historique relativement méconnu en une destination touristique. II va sans dire qu'un tel développement peut causer des conflits avec d'autres types de touristes qui viennent voir la chapelle pour des raisons d'ordre religieux.

On observe le même type de contradiction avec les sites naturels employés dans les films. Le problème principal ici est d'ordre environnemental. Par exemple, le New Zealand Department of Conservation a insisté pour que soient démantelés les décors employés pour le tournage de nombreuses scènes du film The Lord of the Rings afin de protéger les environnements fragiles. Je crois que si la compagnie de production avait été autorisée à conserver ses décors, un conflit aurait inévitablement vu le jour entre les adeptes de la nature et ceux qui y recherchent de la solitude, et le flot potentiel de visiteurs qu'aurait engendré la destination.

Puisque les studios sortent de plus en plus tourner hors studios, il est possible de penser que de plus en plus de destinations chercheront à développer du ciné-tourisme. Ce ne sont pas toutes les attractions qui réussiront.

Qu'est-ce qui peut aider à nourrir la ciné-destination?

Premièrement, les acteurs du tourisme doivent être familiers avec les productions qui ont déjà été réalisées dans une destination. Deuxièmement, ils doivent développer un réseau d'information avec les offices régionaux de cinéma afin d'être informés à temps des projets de films à venir. II suffit ensuite d'étudier les films en regard des lieux de tournages utilisés dans le but d'identifier des pistes potentielles de succès en termes d'attractions ciné-touristiques.

\section{Plusieurs offices de tourisme tentent de vendre leur destination à l'industrie du cinéma. Cette stratégie est-elle vraiment bonne?}

Comme mentionné plus tôt, le secret de la réussite se trouve dans la qualité de la production en question. Le plus grand défi pour les gestionnaires de destination lorsque vient le temps de développer une stratégie de ciné-tourisme est d'être capable d'identifier les ingrédients clés sous-jacents au film ou à la série télévisée. Si tous les ingrédients y sont, cela peut engendrer une bonne couverture médiatique et un bon flot de nouveaux touristes simplement en raison de l'image positive de la destination.

\footnotetext{
Quel est le plus grand défi pour la destination, en ciné-tourisme?

Le plus grand problème vient du fait que la plupart des compagnies de production ne sont tout simplement pas intéressées à travailler avec les gestionnaires des destinations. Elles travaillent avec des budgets et des horaires très serrés. L'équipe de production quitte la région immédiatement le tournage complété. Puis, il y a le problème des droits d'auteur : la plupart des compagnies de production
}

détruisent les décors employés après un tournage. Elles repartent avec leurs biens et, dans plusieurs cas, ne sont pas disposées à laisser à des promoteurs touristiques du matériel comme des images du film ou même la bande-annonce du film à des fins de promotion de la destination. Le troisième grand défi est lié à la qualité des produits de l'expérience ciné-touristique : souvent, il n'y a plus rien sur le lieu de tournage qui nous rappelle la scène qui y a été jouée. Par conséquent, il faut des spécialistes formés en production cinématographique et en gestion du tourisme pour créer des produits de ciné-tourisme qui arriveront à satisfaire les attentes des ciné-touristes.

\section{Les films hollywoodiens occupent une part importante de l'intérêt des ciné-touristes. Voyez-vous un potentiel pour le ciné-tourisme dans les productions d'autres cultures?} Absolument. II existe plusieurs exemples un peu partout dans le monde où des séries télévisées nationales ont fait germer du cinétourisme (en Autriche, en Allemagne, en France, en Australie, en Angleterre, en Suède, etc.). Un autre grand exemple est le cas des productions bollywoodiennes tournées à l'étranger qui engendrent un déplacement énorme de touristes indiens vers des destinations comme l'Autriche, la Suisse ou la Nouvelle-Zélande. La série télévisée sud-coréenne Winter Sonata fut un tel succès au Japon et dans d'autres coins de l'Asie que les principaux lieux de tournage ont été envahis par les touristes.

Quelle taille le succès d'un film ou d'une série télévisée doit-il avoir pour nourrir le ciné-tourisme? Combien de temps une destination peut-elle espérer pouvoir tirer profit du succès d'une production?

J'ai bien peur qu'il n'y ait pas de formule en termes de lien entre le succès au box office et le potentiel touristique. II y a des indications, cependant, à l'effet qu'un film bien reçu du public a de meilleures chances d'engendrer du ciné-tourisme simplement parce que la production prend auprès du public. De là, il n'y a qu'un petit pas pour établir un lien émotif avec les lieux où l'histoire «se déroule». C'est aussi très difficile de prédire la longévité du cycle de vie d'un film : dans plusieurs cas, les films ont un cycle de vie d'environ deux ou trois ans (il en est de même du ciné-tourisme). Voilà pourquoi il ne faut pas sous-estimer l'impact des séries télévisées qui ont un cycle de vie beaucoup plus long. Pensez simplement à la série télévisée américaine Lost et au nombre d'années auxquelles Hawaii a bénéficié du succès de cette série et de sa valeur commerciale pour ses îles.

De plus en plus de films reposent sur les techniques d'animation par ordinateur ou sur le dessin. Ainsi, les décors de film (lieux et paysages) ne sont plus nécessairement "réels". Est-ce que cette transformation du cinéma affectera le ciné-tourisme?

Laissez-moi répondre par un exemple. Les autorités locales de la province chinoise de Hunan ont changé le nom de la montagne Southern Sky Column, dont ont été inspirées les montagnes flottantes Hallelujah, pour maintenant l'appeler Avatar Hallelujah Mountain. Les touristes peuvent maintenant acheter un «Tour magique vers Avatar-Pandora "...

Rendez-vous sur le site Internet de Téoros, pour un second entretien avec J.R. Brent Ritchie, professeur à l'Université de Calgary, à propos de l'excellence des destinations. 